\title{
Integrated Qualitativeness in Design by Multi-Objective Optimization and Interactive Evolutionary Computation
}

\author{
Alexandra Melike Brintrup \\ Cranfield University \\ School of Industrial Manufacturing \\ Science \\ Bedfordshire, MK43 0AL, UK \\ a.brintrup@cranfield.ac.uk
}

\author{
Jeremy Ramsden \\ Cranfield University \\ School of Industrial Manufacturing \\ Science \\ Bedfordshire, MK43 0AL, UK \\ i.ramsden@cranfield.ac.uk
}

\author{
Ashutosh Tiwari \\ Cranfield University \\ School of Industrial Manufacturing \\ Science \\ Bedfordshire, MK43 0AL, UK \\ a.tiwari@cranfield.ac.uk
}

\begin{abstract}
The concept of qualitativeness in design is an important one, and needs to be incorporated in the optimization process for a number of reasons outlined in this paper. Interactive Evolutionary Computation and Fuzzy Systems are two of the widely used approaches for handling qualitativeness in design optimization. This paper classifies the types of qualitativeness observed in design optimization, makes the case for their necessity, and proposes a novel framework for handling them, combining the two approaches in an evolutionary multi-objective optimization platform. Two components of the framework are tested using the floor-planning problem, and observations are reported. Future work is defined on the development of the framework.
\end{abstract}

\section{Introduction to Qualitativeness}

Qualitative is a term often used in opposition to the word quantitative that refers to numerically representable phenomena whose characteristics are universally unchanged. The term qualitative on the other hand refers to phenomena whose characteristics are best expressed by narrations, opinions and beliefs rather than quantities. Qualitative phenomena are subjective by their nature and their characteristics may differ with the domain in which they are handled.

Haack [1] has stressed the necessity of differentiating between uni-dimensional and multi-dimensional qualitativeness. The former refers to qualitative narratives whose conceptual meaning are universally agreed, however the quantitative bounds remain subjective. A simple example is the adjective "Tall", which has a universally uniform conceptual meaning although if the adjective was to be quantified, the bounds would vary depending on the object in question and the domain the object is considered in. Multi-dimensional qualitativeness describes phenomena whose conceptual meaning is subjective. The terms "pretty" or "simple" could exemplify multi-dimensionality in qualitativeness. These types of phenomena are difficult to codify and quantify. The term unidimensional makes a reference to the universally accepted one dimensionality of the phenomena it refers to, whereas the term multi-dimensional can describe a phenomenon by its many qualities. The term "Tall" would have a single length dimension whereas the term pretty could have various dimensions referring to many qualities of the object in consideration.
The two views on supporting subjective multidimensional views in design consist of (1) generalizing the subjective opinion and reflecting a bigger percentage of the population's views, or (2) considering individuality. In today's design world considerable attention is given to satisfy a wide range of customer demands, where methods such as market segmentation are practiced to serve to individual sensitivities. This paper therefore takes on the secondary view for dealing with qualitativeness.

This paper concentrates on the concept of qualitativeness in the domain of evolutionary design optimization and presents a handling mechanism for qualitativeness in evolutionary optimization. The rest of this section looks into types of qualitativeness in this domain, the need for handling the concept of qualitativeness, and real life examples where a formal handling mechanism is needed.

Section 2 briefly reviews previously proposed soft computing approaches for incorporating qualitativeness in evolutionary design optimization, and proposes a novel soft computing based framework to be used to address different type of qualitativeness.

Section 3 presents an illustrative application that has been modeled using two components of the developed framework, and identifies the challenges that the issue of qualitativeness has posed to the optimization process and the framework.

Future work planned to improve the framework is included in Section 4.

\section{Qualitativeness in Evolutionary Multi- Objective Design Optimization}

\subsection{Types of Qualitativeness}

Many real life design problems have multiple objectives, each of which has a different measure and complex relations with other objectives. It is then preferable to obtain a set of optimal solutions each with its own blend of criteria, in order to decide on a compromise solution. Evolutionary computing based platforms have become ideal for handling multiple objectives due to a number of advantages summarized by Deb [2]. The authors have considered the concept of qualitativeness in evolutionary multi-objective design optimization (referred to as EMODO in the rest of this paper), where a given design optimization problem has multiple objectives that are to be 
optimized and the optimization process is handled by evolutionary computing.

The previous section identified two types of qualitative phenomena. In the domain of EMODO, the types of uni and multi-dimensional qualitativeness manifest themselves in the forms of objectives. However, the consideration of qualitative phenomena in EMODO brings on the need for identifying additional qualitative features that such a problem may include.

The authors of this paper have classified preferencebased qualitativeness as an additional type of qualitativeness in the domain of evolutionary design optimization [3]. This type refers to the inclination of a designer towards a selection of design solutions based on his subjective opinion and intuitive judgment. The observed difficulty in verbalizing the reason of choice, as well as the rapidly varying extent of the inclination, has shown the need for a third type of qualitativeness that is not in the form of an objective itself but is nevertheless incorporated in the search. Preferences can be expressed as priori, posteriori or interactively. Different preference handling mechanisms are summarized by Coello [4].

A final qualitative feature in the design optimization problems shows itself in the form of constraint handling. In real life problems constraints can be in various forms, soft constraints that are open to compromise as information on the search space is gathered, hard constraints that are non-negotiable (such as constraints related to safety factors). When soft constraints appear in the optimization process a means of flexible constraint handling should be incorporated. Similar to the preferencebased qualitativeness, qualitative constraints are not objectives themselves, but pose a qualitative affect on the search space.

Figure 1 summarizes the qualitative features that may be present in an evolutionary design optimization process.

In EMODO, multiple objectives of uni or multidimensional types as well as quantitative objectives may need to be pursued. The complexity of the problem at hand

\begin{tabular}{|c|c|c|c|c|}
\hline Domain & Case & $\begin{array}{l}\text { Uni-dimensionally qualitative } \\
\text { objective }\end{array}$ & $\begin{array}{l}\text { Multi-dimensionally qualitative } \\
\text { objective } \\
\text { (Maximizing user satisfaction) }\end{array}$ & Quantitative objective \\
\hline \multirow{2}{*}{$\begin{array}{l}\text { Engineering } \\
\text { Design }\end{array}$} & Piping design & Min. Unit size & $\begin{array}{c}\text { Noise } \\
\text { Vibration } \\
\text { Ergonomics } \\
\text { Customer interface } \\
\end{array}$ & Max. Pumping speed \\
\hline & $\begin{array}{l}\text { Conceptual aircraft } \\
\text { design }\end{array}$ & Min. Flyover noise & $3 \mathrm{D}$ Visualised design concept & Min. Fuel Consumption \\
\hline $\begin{array}{l}\text { Ergonomic } \\
\text { Product } \\
\text { Design } \\
\end{array}$ & $\begin{array}{l}\text { Household product } \\
\text { bottle design }\end{array}$ & Min. Complexity of design & $\begin{array}{l}\text { Ergonomics } \\
\text { Aesthetics }\end{array}$ & Min. Cost of production \\
\hline $\begin{array}{l}\text { System } \\
\text { Design }\end{array}$ & $\begin{array}{l}\text { Heating Appliance } \\
\text { Interface Design }\end{array}$ & $\begin{array}{l}\text { Minimise overall PCB size } \\
\text { Maximize space between } \\
\text { components }\end{array}$ & $\begin{array}{l}\text { Simplicity of interface } \\
\text { Ergonomic concerns }\end{array}$ & $\begin{array}{c}\text { Minimize temperature/heat } \\
\text { distribution } \\
\text { Minimise assembly time }\end{array}$ \\
\hline \multirow{2}{*}{$\begin{array}{l}\text { Architectural } \\
\text { Design }\end{array}$} & Kitchen Design & Max. Size of free area & $\begin{array}{l}\text { Various aesthetic concerns such as } \\
\text { flow of layout }\end{array}$ & Min. Cost of build \\
\hline & House Floor Planning & $\begin{array}{l}\text { Max. Sizes of a number of } \\
\text { rooms given a constant } \\
\text { bounding area }\end{array}$ & $\begin{array}{l}\text { Various aesthetic concerns such as } \\
\text { flow of layout }\end{array}$ & Min. Cost of build \\
\hline
\end{tabular}

Table 1. Examples of design objectives

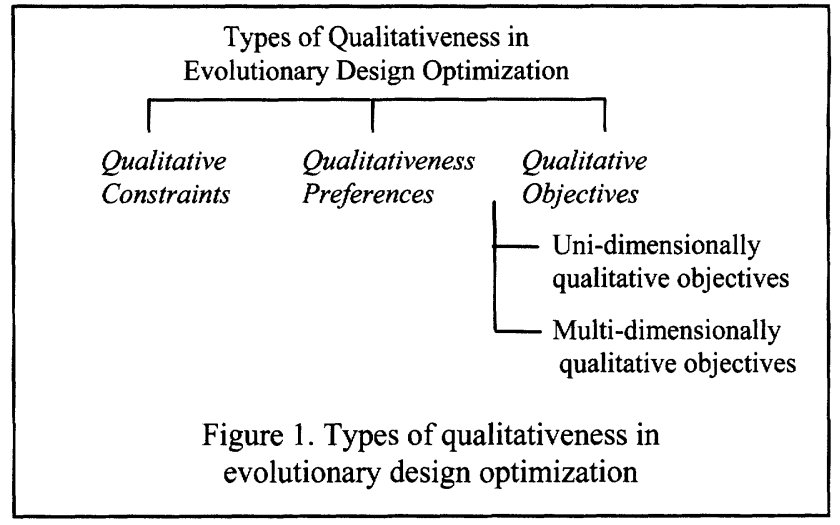

increases with the existence of qualitative preferences and constraints on quantitative objectives as well as qualitative objectives. Table 1 illustrates typical design optimization examples with objectives varying in nature.

\subsection{On the need for Qualitativeness}

The need for handling qualitativeness in design optimization have been identified through a literature survey and interviews with design practitioners, as follows:

Taking advantage of experience and judgment: When solving complex design problems, designers influence how the problem is defined and solved depending on their individual experience accumulated over the years. The influence is qualitative in nature and constitutes an essential aspect of problem solving. Even in highly quantitative problems where the qualitative influence does not manifest itself, as strongly as an objective by itself, the qualitative influence still remains a reality and can manifest itself in terms of solution preferences or constraints on the search space.

Completing the problem definition: It has been reported that there may be cases where an accurate numerical representation of search space is not readily available [5]. 
In this case, qualitative information can close the loop of problem definition by taking advantage of human approximate reasoning and qualitative judgment. The capture of qualitative information can improve problem definition and allow faster optimization in the next design iteration.

Dealing with various design views: A given engineering design has many views in real world applications. These may typically include marketing, engineering and quality control each with its own definition of what constitutes a successful design. The formal representation of these views by multiple qualitative and quantitative objectives can improve the information flow between different views, as their implications on other views are better and faster understood and lead to a more informed compromise decision making. Additionally, incorporating different views can allow better conceptual experimentation across the solution search space and can lead to innovative solution discovery.

Dealing with the re-definition of objectives:

Often a complete definition of the optimization problem shapes up during the information gathering stage, where features of possible solutions are observed. The redefinition process may include reconsideration of constraints. With the discovery of new solution properties, relative importance of objectives may move the search to areas that were initially thought unsuitable. A means of flexible handling of problem redefinition can be provided if the framework used allows qualitative considerations to be satisfactorily included.

\section{A New Soft Computing Framework for Handling Qualitativeness}

\subsection{A Review on Soft Computing Based Frameworks for Handling Qualitativeness in EMODO}

The need for suitable techniques for handling qualitativeness in EMODO steers the search towards the field of soft computing.

The authors have presented a comparative analysis between Fuzzy Systems (FS) and Interactive Evolutionary Computing (IEC) and summarized the features of previously proposed frameworks that incorporate qualitativeness in multi-objective optimization [3].

In summary, Oduguwa et al. [5] has proposed modeling, and hence quantifying, qualitative objectives using FS and creating the problem as a multi-objective one. Parmee and Cvetkovic [6], has modeled designer preferences using FS and proposed an agent based negotiation system. Shibuya et al. [7] applied IEC to multi-objective optimization in the field of animation design, allowing the user to give subjective ratings from each objective's point of view, and more recently, Kamalian et al. [8] has used IEC on micro electrical mechanical system (MEMS) design, to include subjective influence on a primarily quantitative multi-objective problem.

Although a relatively higher number of studies have been carried out on preference incorporation in multi- objective quantitative problems [4], and application of IEC to optimize designs based solely on subjective ratings [9], there is limited work reported on treating qualitative objectives of a design problem in a multi-objective problem.

Both Oduguwa et al. and Parmee et al. have utilised FSM to convert unidimensional qualitative criteria into quantitative criteria for inclusion in a multi-objective optimisation framework. In both of these approaches, multidimensional qualitative data has not been handled. In Kamalian et al.'s MEMS design a sequential approach was taken. The MOGA (Multi-objective Genetic Algorithm [10]) was run for a given number of generations before users gave preference scores to the last generation using the IEC, and evolved the designs further. Shibuya et al. employed a similar principle where the final generation of non-dominated solutions are presented to the user who sorts them in order of preference. These ratings were then used by the IEC to generate a new population. Both Shibuya et al. and Kamalian et al. used MOGA as their platform.

The approach taken in this paper uses the user ratings as an objective's fitness value and continuous interaction with the user is pursued. Furthermore, the approach takes into account both multidimensional and unidimensional qualitativeness. The evolutionary multi-objective optimization algorithm used is NSGA 2, by Deb et al. [11]. The following section explains the proposed framework further.

\subsection{Towards the Development of a new Soft Computing Optimization Framework}

Given the different types and the needs of qualitativeness identified for its flexible accommodation, the authors propose a soft computing based framework to handle qualitativeness in EMODO.

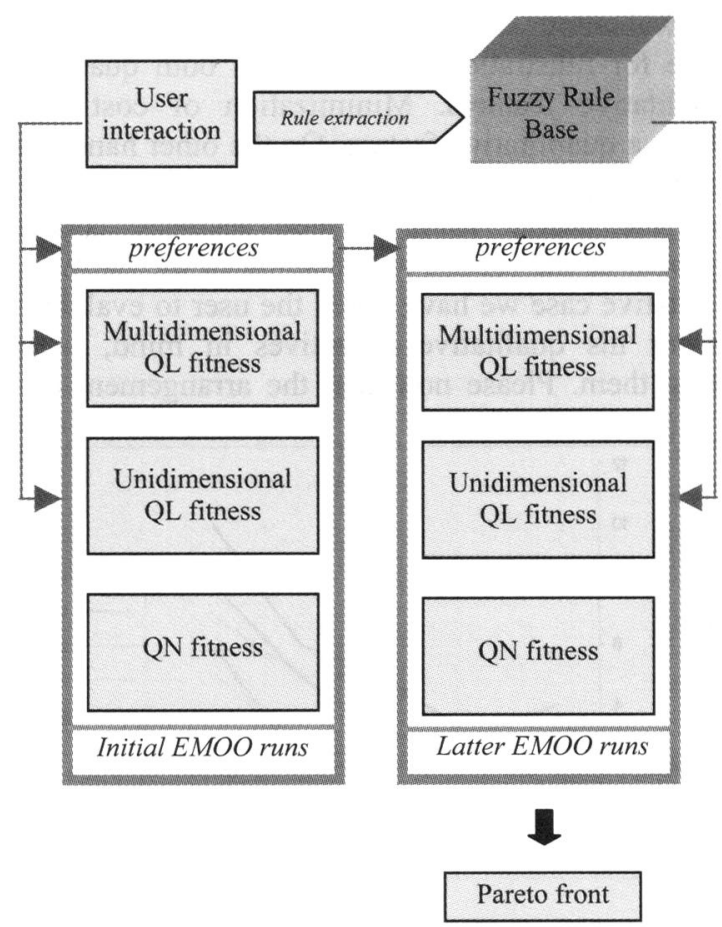

Figure 2. IMODO Framework 
Figure 2 illustrates the components of the proposed framework, named as IMODO (Interactive Multi-objective Design Optimization).

Interactive Genetic Algorithm (IGA) component: The evaluation of multi-dimensionally qualitative objectives is permanently outsourced to the designer. This component also initially handles uni-dimensionally qualitative objectives until sufficient fuzzy rule extraction is obtained.

Fuzzy Rule Base (FRB) component: A background fuzzy rule extraction process is introduced during the designer-machine interaction. This component captures qualitative design intent by relating the qualitative ratings to features of the design.

Evolutionary Multi-objective Optimization (EMOO) component: The EMOO component optimizes qualitative and quantitative objectives together.

The designer can dynamically change constraints and preferences between objectives and the FRB remains visible to the designer for modification. This allows continuous improvement of the problem definition as a result of search space information gathering. Designers can modify solutions and reinsert into the system and freeze different features of the design at hand. This is intended to accelerate the convergence to satisfactory solutions.

\section{Illustrative Case - House Floor Planning}

\subsection{Definition of Problem}

The IGA and EMOO components of the IMODO framework were applied to the house floor-planning problem [12].

The goals are to find the width and length of each room as shown on figure 3 that will (1) minimize the cost of build subject to some constraints, and (2) maximize subjective user evaluation received by the interactive fitness evaluation element. This problem is an ideal candidate for illustration as it includes both quantitative and qualitative features. Minimization of cost clearly constitutes a quantitative feature. On the other hand multidimensional qualitative features, that the user might evaluate the design from, include aesthetic concerns, arrangement of the rooms, and shaping of the hallway. In the qualitative case we have asked the user to evaluate the design for his qualitative objectives in mind, without localizing them. Please note that the arrangement of the

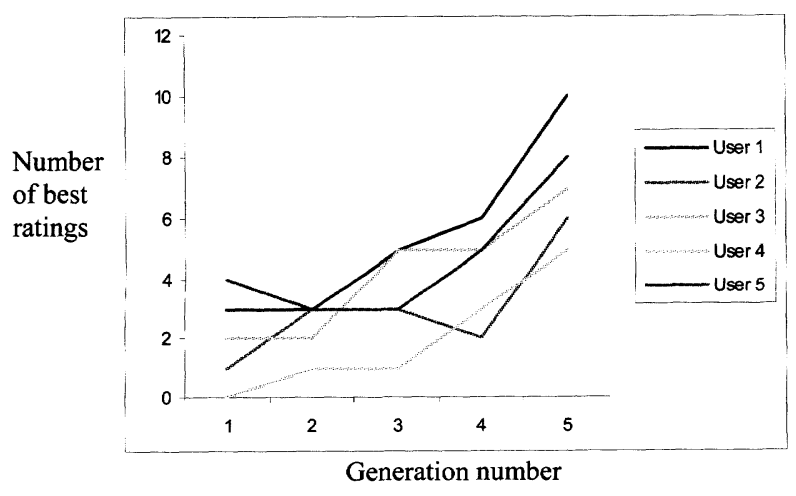

Figure 4. User satisfaction over 5 generations rooms has been added to the original problem in [12] in order to include an additional qualitative aspect to the problem.

Although at first the problem appears to consist of 14 dimensional parameters referring to the width and length of each room, analysis of the problem reveals that some parameters can be computed from others. The dimensional parameters of the rooms are shown on figure 5, along with the constraints. The labels for each room are encoded after the dimensional parameters. Real valued encoding has been used. Cost of each room is the same except the kitchen and the bathroom. All rooms except the hall should be of rectangular shape.

\section{2 Setup and Experiments}

The interactive evolutionary design process is outlined on figure 5 .

Population size of 12 , a mutation probability of 0.01 and one-point crossover with a probability of 0.95 have been used. Simulated Binary Crossover with a distribution index of 20 and real mutation with a distribution index of 10 were used. The EMOO algorithm used is the elitist NSGA 2 [11] with crowded tournament operator.

The subjective rating was taken between 1-10, with 1 showing highest user satisfaction.

Subjective tests were conducted on three students from Cranfield University. The starting population was filled with random individuals. Users continued to run the program until either the results were satisfactory or generation 10 was reached. Each user conducted two tests. Table 2 shows the number of generations until a satisfactory declared result was reached for each user while figure 4 shows the number of ratings below 4 for each generation for each user trial. Figure 6 displays the convergence of qualitative objective vs. quantitative objective over 5 generations.

\begin{tabular}{|c|c|}
\hline \multirow{2}{*}{ User } & $\begin{array}{c}\text { Number of generations } \\
\text { for satisfactory declared } \\
\text { result }\end{array}$ \\
\hline \multirow{2}{*}{2} & 5 \\
\cline { 2 - 2 } & 6 \\
\hline \multirow{2}{*}{3} & 7 \\
\cline { 2 - 2 } & 4 \\
\hline \multirow{2}{*}{4} & 10 \\
\cline { 2 - 2 } & 10 \\
\hline \multirow{2}{*}{5} & 6 \\
\hline
\end{tabular}

Table 2. Test results of user with satisfaction IMODO framework

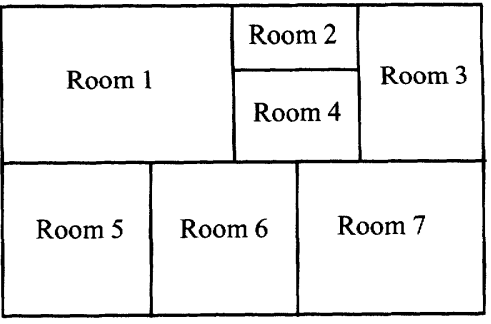

Figure 3. Floor-planning problem 


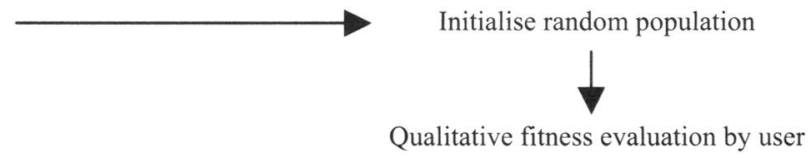

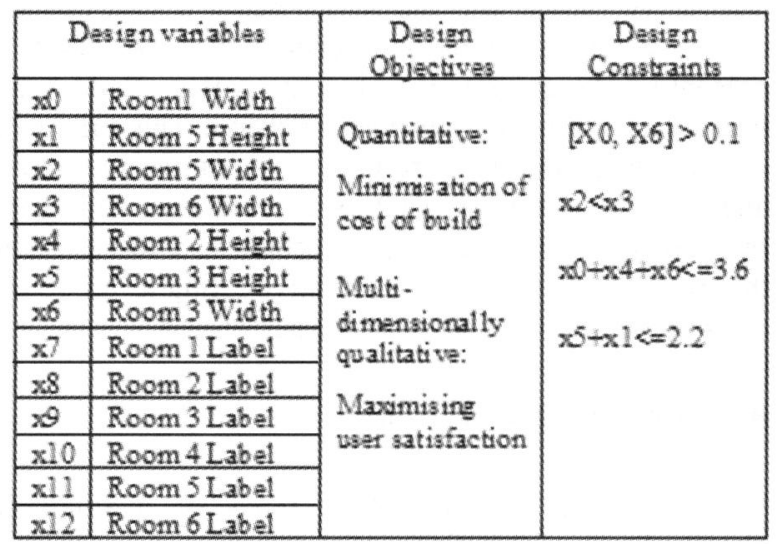

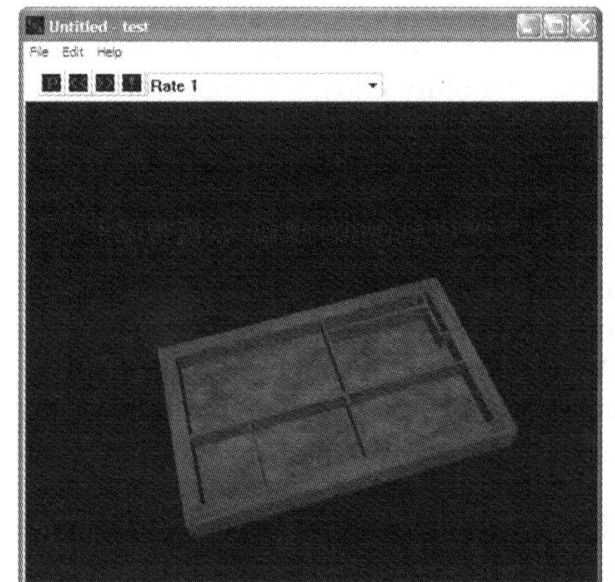

Quantitative fitness evaluation of individuals in population
Qualitative fitness rating

\begin{tabular}{l}
\hline $\begin{array}{l}\text { Rotation and } \\
\text { Translation } \\
\text { controls }\end{array}$ \\
\hline $\begin{array}{l}\text { Labelling for each } \\
\text { room }\end{array}$ \\
\hline
\end{tabular}

\section{Editing}

properties of GA before each run is allowed

Direct

chromosome manipulation

Figure 5. Outline of the interactive evolutionary design process

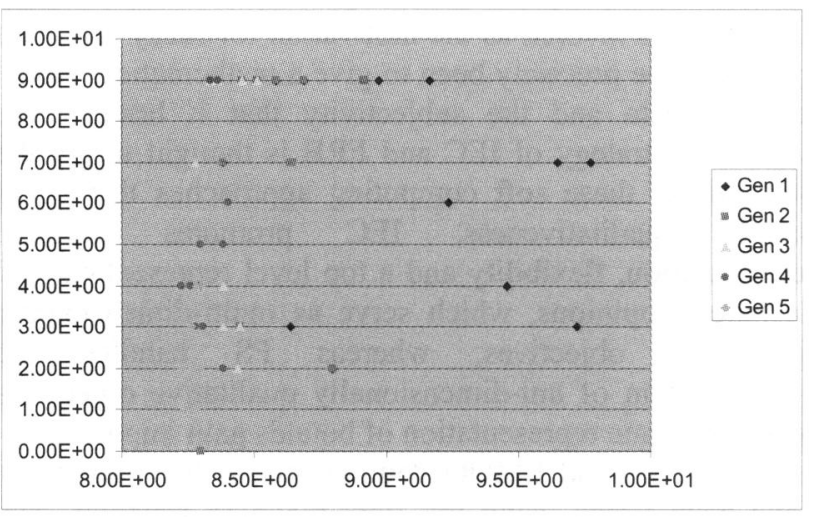

Figure 6. Convergence of qualitative objective (y axis) vs. quantitative objective ( $\mathrm{x}$ axis) over 5 generations

\subsection{Discussion of Results}

The initial results of the IMODO framework showed convergence to satisfactory results within reasonable number of generations. Pursuing satisfaction of two objectives of different nature in a single framework proved achievable and satisfactory. However, with only 5 nonexpert users participating in the test, it is has not been able to draw statistically significant results on whether the IMODO framework has resulted in successful user satisfaction in both types of objectives. It is necessary to further test the framework with a higher number of users and with relevant experts from the problem domain. It is also planned to test the framework where the users vocalize their qualitative objectives and pursue the evaluation with only these objectives in mind. At the moment multi-dimensional qualitative objectives such aesthetics of the layout or room arrangements have been tested; so it is planned to pursue the tests with a unidimensional qualitative objective such as targeting maximum / minimum sizes of a number of rooms. Further developments on the framework itself are planned such as outsourcing of uni-dimensional qualitative objectives to the fuzzy evaluation and including preference-handling mechanisms, in order to address other types of qualitativeness outlined in section 2.1.

The following are observations in dealing with qualitative and quantitative objectives as a multi-objective problem are reported:

Scalability: The qualitative fitness evaluation is a discrete rating and can present scalability issues when the quantitative objective function is continuous where the Pareto front shows a discrete dimension against a continuous dimension.

Human fatigue: The IGA approach of blackbox fitness evaluation by the user is extremely versatile in handling changing definitions of uni and multi-dimensionally qualitative objectives since no effort for reconfiguration is necessary. As the user modifies individuals and reinserts into the population, as well as gaining information about the behavior of the multi-objective search space, the IGA becomes a two way informative process and this accelerates convergence when compared with the nonexistence of this feature. On the other hand, human fatigue prevents the evaluation of a large number of 
generations or large populations by the human user. Research on various methods of dealing with fatigue in IEC is conducted at the moment. One of these promising methods, which might be suitable for the IMODO framework, includes Euclidean distance calculation [13], where the differences in features of design solutions are calculated. This is then used for clustering the solutions. After this process one randomly chosen design from each cluster is shown to the user for rating, and the same rating is applied to the whole cluster the design belongs to. A main disadvantage with this method could be, the possibility of missing out on subjectively exceptional designs. The calculation of distances and clustering of individuals therefore has to be performed with care.

Granularity: A considerable change in quantitative space does not necessarily have a considerable impact on qualitative space, which makes the human perception of change in the qualitative space difficult. As the convergence reaches to an optimum it becomes difficult for a human to evaluate solutions with very minor differences. One method of handling this issue is currently considered by the authors as follows: In order to validate the similarity of designs at a given generation number Euclidean distance calculation can be used, and only those designs with high differences are shown to the user. After reaching a set minimum distance, the weighting of the quantitative objective is gradually increased. This takes the burden off the user who otherwise needs to rate visually similar designs with quantitative differences.

An additional observation related to this included that as population converges the differences between ratings decrease, when designs are looked from the whole perspective (comparison with the whole range of designs seen until current generation). If only the current generation is considered however, the difference between designs have a higher impact on the differences of ratings from each other.

User inconsistency: When using IEC we cannot guarantee a mathematically consistent rating that will impact in proportion or accordance with the changes in the qualitative space. The reasons for this might be simply due to user inconsistency or human fatigue, such as the user not remembering older designs for an accurate comparison, or not being able to provide a rating that can accurately represent this comparison in terms of scaling. To minimize the effect of this issue, a user inconsistency detection method and a way of dealing with inconsistency needs to be implemented. Current thoughts include implementing a simple strategy such as the following:

(1) Compare [distance between designs $\mathrm{A}$ and $\mathrm{B}$ ] with [rating difference of designs $\mathrm{A}$ and $\mathrm{B}$ ],

(2) If the difference exceed a given inconsistency threshold, increase weighting of the quantitative objective.

This method shifts the importance of the objective so as not to let the population sacrifice from the quantitative objective as a result of the inconsistency. The number of the generation the inconsistency occurs has to be carefully taken into account as it has been observed that initial generations are an information-gathering period for the user and hence where inconsistency is likely to occur.

Additional to these observations, it has been debated whether the quantitative objective should be shown to the user with each individual. This might introduce bias to the qualitative rating, and the rating being a sole qualitative objective rating might be arguable. On the other hand presenting the quantitative objective value would help the user making a better informed decision. This might be an option when there is more than one quantitative objective and the user rating is used as a preference rating rather than an objective in itself. Nevertheless a test comparing the two options (usage of qualitative rating as an objective or as preference rating) has to be performed.

\section{Concluding Remarks}

This paper outlined three types of qualitativeness observed in EMODO applications, as Qualitative objectives, constraints and preferences. The qualitative objectives include multi and uni-dimensionally qualitative objectives. The case for handling qualitative needs of designers is made by showing real life design examples and outlining the findings of a literature survey and interviews with design practitioners. The authors then proposed a soft computing based framework for its handling utilizing two tools that are widely used to handle qualitativeness: Fuzzy Systems and IGA. The framework pursues qualitative objectives together with quantitative objectives in an EMOO algorithm. Outsourcing of unidimensional qualitative objectives to the fuzzy evaluation is favored as the motivation for fuzzy rule base systems have precisely been to give a mathematical model to such data and the subjectivity that it brings. The combined strategy of IEC and FRB is thought to get the best out of these soft computing approaches formed to handle qualitativeness. IEC promotes problem reformulation, flexibility and a top level representation of designer's opinions, which serve as multi-dimensionally qualitative objectives, whereas FS handles the quantification of uni-dimensionally qualitative objectives where accurate representation of bounds gain importance.

The IGA and EMOO components of the framework have been tested using the floor-planning problem. The findings reported include challenges observed in the areas of scalability, human fatigue, granularity and user inconsistency. Despite the observed challenges of incorporating qualitativeness in EMODO by the proposed framework, the overall strategy seems to give satisfactory results and satisfy the needs outlined in Section 1.3.

The next steps of development will include (1) the addition of fuzzy rule base in an attempt to reduce the burden of the designer and (2) incorporation of preference handling mechanisms. The performance of different EMOO algorithms will also be evaluated. 


\section{References}

[1] S. Haack, Deviant Logic, Fuzzy Logic, Beyond the Formalism, Chicago and London: The University of Chicago Press, 1996, pp. 9

[2] K. Deb, Multi-objective Optimization Using Evolutionary Algorithms, Wile Interscience series in Systems and Optimization, Advisory Ed. S. Ross, R. Weber, ISBN 047187339X, 2001

[3] A. Brintrup, A. Tiwari , J. Gao, "Handling Qualitativeness in Evolutionary Multiple Objective Engineering Design Optimization", Proc. of International Conference of Computational Intelligence, Vol.1, pp.236-240, December 2004

[4] C. C. Coello, "Handling Preferences in Evolutionary Multiobjective Optimization: A Survey", Proc. of the Congress on Evolutionary Computation, July 2000, Piscataway, New Jersey,Vol. 1, pp. 30-37

[5] V. Oduguwa, A. Tiwari, R. Roy, "Handling Integrated Quantitative and Qualitative Search Space in a Real World Optimization Problem", Congress on Evolutionary Computation (CEC), Canberra, Australia, 2003, pp.1222-1229

[6] I. C. Parmee, D. Cvetkovic, A. Watson, C. Bonham, "Multi-Objective Satisfaction within an Interactive Evolutionary Design Environment," Journal of Evolutionary Computation, vol. 8, No. 2, MIT Press, pp. $197-222,2000$

[7] M. Shibuya, H. Kita, S. Kobayashi, "Integration of Multi-objective and Interactive Genetic algorithms and its application to Animation Design", Proc. Of IEEE Systems, Man and Cybernetics (SMC), Tokyo, Japan, 1999, vol. 3, pp. 646-651

[8] R. Kamalian, H. Takagi, A. Agogino, "Optimized Design of MEMS by Evolutionary Multi-Objective Optimization with Interactive Evolutionary Computation", Genetic and Evolutionary Computation Conference (GECCO), Seattle, US, 2004, pp. 1030-1041

[9] H. Takagi, "Interactive Evolutionary Computation: Fusion of the capabilities of EC Computation and Human Evaluation", Proc. of the IEEE, Vol.89, No.9, 2001, pp.1275-1296

[10]C. M. Fonseca, P. J. Fleming, “Genetic Algorithms in Multiple Objective Optimisation: Formulation, Discussion, and Generalization", Proc. of the Fifth International Conference on Genetic Algorithms, Los Altos CA, 1993, Vol. 26, pp.416-423
[11]K. Deb, S. Agrawal, A. Pratap, T. Meyarivan, " $A$ Fast Elitist Non Dominated Sorting Genetic Algorithm for Multi Objective Optimization: NSGA-2", Proceedings of Parallel Problem Solving from Nature (PPSN) $6^{\text {th }}$ International conference, Paris, France, 2000, pp. 858862

[12]A. D. Radford and J. S. Gero, Design Optimization in Architecture, and Building, Van Nostrand Reinhold, New York

[13] K. Nishio, M. Murakami, E. Mizutani, N. Honda, Fuzzy Fitness Assignment in an Interactive Genetic Algorithm for a Cartoon Face Search, Genetic Algorithm and Fuzzy Logic Systems: Soft Computing Perspectives, Eds. E. Sanchez, T. Shibata, L. Zadeh, World Scientific, 1997 